\title{
A sensitivity analysis of the standard deviation of the co-polarized phase difference for sea oil slick observation
}

\author{
Andrea Buono, Student Member, IEEE, Ferdinando Nunziata, Senior Member, IEEE, Carina Regina de \\ Macedo, Student Member, IEEE, Domenico Velotto, Member, IEEE, and Maurizio Migliaccio, \\ Fellow Member, IEEE
}

\begin{abstract}
In this study, a time series of 33 TerraSARX co-polarized Synthetic Aperture Radar (SAR) imagery collected in Stripmap mode over the Gulf of Mexico in a wide range of incidence angles and sea state conditions, is exploited, together with a theoretical framework based on the X-Bragg scattering model, to analyze the effects of noise, angle of incidence (AOI) and wind speed on the standard deviation of the co-polarized phase difference $\left(\sigma_{\phi_{c}}\right)$ evaluated over sea surface with and without oil slicks. This large dataset represents an unprecedented opportunity to analyze, for the first time, the influence of both SAR acquisition and surface parameters on the broadening of the co-polarized phase difference probability density function (pdf), $p_{\phi_{c}}\left(\phi_{c}\right)$.

Experimental results show that the $\mathrm{X}$-Bragg scattering model, here adopted to predict the sea surface $p_{\phi_{c}}\left(\phi_{c}\right)$, gives an understanding of the increasing trend of $\sigma_{\phi_{c}}$ with respect to AOI. It is shown that the noise significantly contributes to broaden $p_{\phi_{c}}\left(\phi_{c}\right)$ over both slick-free and slick-covered sea surface, while the effects of low-tomoderate wind regimes are negligible. In addition, $\sigma_{\phi_{c}}$ exhibits a larger sensitivity to the scene variability, if compared to single-polarization intensity channels, over both slick-free and oil-covered sea surface. This sensitivity is more pronounced at lower AOIs due to the higher noise equivalent sigma zero (NESZ) that affects larger AOIs.
\end{abstract}

\section{INTRODUCTION}

A systematic mapping of sea oil slicks is a challenging task which is of paramount importance to both preserve the environment and support oil exploration and extraction activities. Nonetheless, high-resolution spaceborne remote sensing instruments represent a technological and cost-effective solution to accomplish this task [1]. Within this context, Synthetic Aperture Radar (SAR) is a key tool for sea oil slick observation due to

Andrea Buono, Ferdinando Nunziata, Carina Regina de Macedo, and Maurizio Migliaccio are with the Dipartimento di Ingegneria, Università degli Studi di Napoli Parthenope, Napoli, Italy e-mail: \{andrea.buono, carinaregina.demacedo, ferdinando.nunziata, maurizio.migliaccio\}@uniparthenope.it

Domenico Velotto is with the Remote Sensing Technology Institute, German Aerospace Center (DLR), 28199 Bremen, Germany e-mail: Domenico.Velotto@dlr.de

Manuscript received xx revised . its almost all-weather and fine spatial resolution imaging capabilities [1]-[4]. SAR-based sea oil slick observation is grounded on the fact that the presence of an oil layer over the sea surface reduces the short Bragg resonant waves and, therefore, generates a low backscatter area which appears, in conventional gray-tones SAR imagery, as a homogeneous patch darker than the background sea [5], [6].

Nowadays, there is wide consensus on the extra-benefits provided by polarimetric SAR data for a wide range of marine and maritime applications, including coastline extraction [7], [8], metallic target detection [9], [10] and sea oil slick monitoring [11]-[18]. In the framework of SAR-based sea oil slick observation it was shown that, once an electromagnetic model is available, polarimetric measurements can be successfully used to distinguish actual oil slicks from weak-damping look-alikes and to perform a rough zooning of the damping properties of the observed surfactant [11]-[13].

Sea oil slick observation using SAR imagery is actually a quite mature application whose performance is significantly affected by the amount of scattering information available that, in turn, depends on both sensor's and environmental parameters, e.g.; polarization, angle of incidence (AOI), noise equivalent sigma zero (NESZ), sea state conditions and oil's damping properties [3], [11]. However, even though operational sea oil slick observation is mainly based on single-polarization intensity measurements due to large data availability and the wide area coverage they offer, the key role played by polarimetric information is well-established [1], [2]. The idea that underpins polarimetric SAR-based approaches is to exploit the different scattering properties that characterize slick-free sea surface or weak-damping surfactants and oil slicks [3], [11], [12]. Several polarimetric features have been proposed that allow measuring the departure from the almost deterministic sea surface Bragg scattering which applies over slick-covered sea surface [11], [17]. The performance of those features depend on: i) SAR acquisition parameters, i. e., AOI, incident wavelength and NESZ; ii) sea state conditions, 
i. e., the level of surface roughness and iii) damping properties of the surfactant.

Among the different polarimetric features, the standard deviation of the co-polarized phase difference $\left(\sigma_{\phi_{c}}\right)$, that is linked to the complex correlation between copolarized channels, has been shown to be a reliable and robust, i. e., effective at variance of frequency (L-, $\mathrm{C}-$ and $\mathrm{X}$-band), almost independent of the estimation window size and unbiased when low correlation of copolarized channels applies, estimator of the oil slick departure from Bragg scattering [18]-[20].

In [21]-[23] it was shown that $\phi_{c}$ probability density function (pdf), $p_{\phi_{c}}\left(\phi_{c}\right)$, resembles a Gaussian bell whose mean, $\bar{\phi}_{c}$, is close to $0^{\circ}$ and whose standard deviation, $\sigma_{\phi_{c}}$, depends on the degree of correlation between the co-polarized channels, $\rho$, and on the number of looks. Given the number of looks, $\phi_{c}$ pdf is uniformly distributed when $\rho$ tends to 0 , while it behaves as a Dirac delta function when $\rho$ tends to 1 [23], [24]. It was also demonstrated that, according to the Bragg scattering theory, $\bar{\phi}_{c}$ increases with AOI and incident wavelength, e. g., $\bar{\phi}_{c}$ does not exceed $4^{\circ}$ at $\mathrm{X}$-band for AOI $=60^{\circ}$ [25]. According to Bragg scattering, co-polarized channels are highly correlated and cross-polarized backscattering is almost negligible. This implies that $\sigma_{\phi_{c}}$ is close to $0^{\circ}$. Significant depolarization occurs when dealing with oilcovered sea surface that implies larger $\sigma_{\phi_{c}}$ values [18][20].

In the framework of sea oil slick observation, $\sigma_{\phi_{c}}$ has been recognized to be very effective in both observing oil slicks and discriminating them from weak-damping surfactants [9], [18], [20]. The key result is that $\sigma_{\phi_{c}}$ is able to emphasize the oil slick with respect to the background sea while de-emphasizing the weak-damping surfactant. However, the behavior of $\sigma_{\phi_{c}}$ is found to be significantly affected by AOI, NESZ and meteo-marine conditions [18]-[20].

In this study, a polarimetric analysis is undertaken to investigate the behavior of $\sigma_{\phi_{c}}$ with respect to AOI, NESZ and sea state conditions. The proposed analysis is performed from both theoretical and experimental viewpoint. In particular, a theoretical framework based on the well-known X-Bragg scattering model is exploited to predict the $\sigma_{\phi_{c}}$ behavior with respect to AOI. This theoretical analysis is exploited to give a better understanding on the role played by sensor's and scene parameters on the broadening of $p_{\phi_{c}}\left(\phi_{c}\right)$. Then, an experimental analysis based on a time series of dualpolarimetric HH-VV TerraSAR-X (TSX) SAR scenes, collected in a wide range of AOI and under low-tomoderate wind conditions, is performed. This analysis points out the effects of SAR acquisition parameters, i. e., AOI and NESZ, and environmental parameters, i. e., meteo-marine conditions, on the $\sigma_{\phi_{c}}$ behavior over sea surface and oil slicks. With respect to oil slicks, reference is made to the well-known oil seep related to the Taylor Energy platform accident occurred in the Gulf of Mexico in 2004 [26]. This SAR dataset represents an unprecedented opportunity to undertake, for the first time, a sensitivity analysis of the aforementioned factors on $\sigma_{\phi_{c}}$ evaluated over sea surface with and without oil slicks.

In addition, the sensitivity of the so-called Pauli phase [27] to the presence of oil slicks over the sea surface is also experimentally analyzed, showing that it does not exhibit any sensitivity to sea oil slicks.

The remainder of this paper is organized as follows: the theoretical background is described in section II, while simulation setup and numerical results are detailed in section III; the study area and the SAR dataset are presented in section IV, while experimental results obtained on actual SAR measurements are presented and discussed in section V; conclusions are drawn in section VI.

\section{TheORETICAL BACKGROUND}

In this section, the polarimetric characterization of sea surface backscattering is presented and the relationship between co-polarized phase difference and sensor/surface parameters is discussed.

In order to completely describe the polarimetric scattering from a distributed depolarizing natural scene, second-order field descriptors are needed, i. e., the covariance or coherency matrix $(\mathbf{C} / \mathbf{T})$ must be used [28]. In this study, the covariance matrix formalism is adopted that, in the monostatic backscattering case and under reciprocity assumption, can be expressed as [28], [29]:

$$
\mathbf{C}=\left(\begin{array}{ccc}
\left\langle\left|S_{h h}\right|^{2}\right\rangle & \sqrt{2}\left\langle S_{h h} S_{h v}{ }^{*}\right\rangle & \left\langle S_{h h} S_{v v}{ }^{*}\right\rangle \\
\sqrt{2}\left\langle S_{h v} S_{h h^{*}}\right\rangle & 2\left\langle\left|S_{h v}\right|^{2}\right\rangle & \sqrt{2}\left\langle S_{h v} S_{v v}{ }^{*}\right\rangle \\
\left\langle S_{v v} S_{h h}{ }^{*}\right\rangle & \sqrt{2}\left\langle S_{v v} S_{h v}{ }^{*}\right\rangle & \left\langle\left|S_{v v}\right|^{2}\right\rangle
\end{array}\right)
$$

where $S_{p q}$ is the complex scattering amplitude with $\{p, q\}=\{h, v\}$, while $\langle\cdot\rangle,|\cdot|$ and ${ }^{*}$ stand for ensemble average, modulus and complex conjugate operators, respectively. In this study, the X-Bragg scattering model is adopted to describe polarimetric scattering from sea surface under low-to-moderate wind conditions and at intermediate AOI. The $\mathrm{X}$-Bragg surface scattering model was first proposed and validated using L-band SAR data in [27] to predict the backscattering from slightly rough land surfaces for soil moisture estimation purposes. Then, it was specialized to model sea surface backscattering in [16], [30], [31]. In [16], the X-Bragg scattering model was successfully adopted for sea oil slick observation purposes against $\mathrm{L}-$ and $\mathrm{C}-$ band polarimetric SAR measurements while, in [30], it was employed to detect ships and oil spills from $\mathrm{C}$ - and 
$\mathrm{X}$-band polarimetric SAR imagery.

The X-Bragg scattering model represents an improved version of the Bragg scattering theory that takes into account non negligible cross-polarized backscattering and depolarization effects. The sea is modeled as a reflection symmetric depolarizing surface whose random roughness is introduced by a rotation of the covariance matrix in a plane orthogonal to the scattering one. Accordingly, the sea surface covariance matrix can be predicted, under intermediate AOIs and low-tomoderate sea state conditions, as follows [28], [29]:

$$
\mathbf{C}_{\mathbf{X}}=\left(\begin{array}{ccc}
C_{X_{11}} & C_{X_{12}} & C_{X_{13}} \\
C_{X_{12}} * & C_{X_{22}} & C_{X_{23}} \\
C_{X_{13}} & C_{X_{23}} & C_{X_{33}}
\end{array}\right)=\mathbf{U T}_{\mathbf{X}} \mathbf{U}^{-1},
$$

where the subscript " $\mathrm{X}$ " stands for $\mathrm{X}$-Bragg model, and the special unitary matrix, $\mathbf{U}$, and the matrix $\mathbf{T}_{\mathbf{X}}$ are given in eq. (3) [30], [31]. In eq. (3), $B_{c p}$ and $B_{c m}$ are combinations of the complex Bragg scattering coefficients, while $s_{2 \beta}$ and $s_{4 \beta}$ are functions of the width of the local surface tilting angle, $\beta$, pdf [27].

The parameters $s_{2 \beta}$ and $s_{4 \beta}$ are related to the amount of surface roughness, with $\beta$ that controls both the crosspolarized backscattered power and the co-polarized coherence; while the Bragg scattering coefficients depend on AOI and sea relative permittivity, $\epsilon$ [27]. The polarization-dependent Bragg scattering coefficients are defined as follows:

$$
\begin{gathered}
B_{h h}=\frac{\cos (\mathrm{AOI})-\sqrt{\epsilon-\sin ^{2}(\mathrm{AOI})}}{\cos (\mathrm{AOI})+\sqrt{\epsilon-\sin ^{2}(\mathrm{AOI})}} \\
B_{v v}=\frac{(\epsilon-1)\left(\sin ^{2}(\mathrm{AOI})-\epsilon\left(1+\sin ^{2}(\mathrm{AOI})\right)\right.}{\left(\epsilon \cos (\mathrm{AOI})+\sqrt{\epsilon-\sin ^{2}(\mathrm{AOI})}\right)^{2}} .
\end{gathered}
$$

Note that, according to the Bragg scattering theory, sea surface backscattering is well-predicted by the $\mathrm{X}$ Bragg model when AOI ranges in the interval $20^{\circ}-$ $60^{\circ}$, while the surface tilting angle is assumed to be uniformly distributed in the range $-90^{\circ}-+90^{\circ}$ [27]. This means that the probability of obtaining a smooth sea surface characterized by almost unitary co-polarized coherence and negligible cross-polarized backscattering, i. e., $\beta \approx 0^{\circ}$, is the same of obtaining a rougher sea surface that calls for almost zero co-polarized coherence and significant cross-polarized backscattering, i. e., $\beta$ $\approx 90^{\circ}$ [27]. Nevertheless, it must be noted that, when dealing with actual sea surface SAR measurements, the estimated $\beta$ values typically do not exceed $\pm 30^{\circ}$. This results in a co-polarized coherence larger than 0.95 and a cross-polarized backscattering which is less than $20 \%$ of the total backscattered power when AOI $=40^{\circ}$ [27],
[31].

According to the $\mathrm{X}$-Bragg scattering model,the phase difference between the co-polarized channels, $\phi_{c}$, is predicted using eq. (5) where $L$ and $\Im$ stand for phase and imaginary part, respectively. It can be noted that $\phi_{c}$ depends explicitly on sea surface dielectric and geometric properties, i. e., $\epsilon$ and local tilting, through Bragg scattering coefficients and $\beta$, and on SAR acquisition parameters, i. e., AOI and incident wavelength, through Bragg scattering coefficients. Eq. (5) explicitly points out that both real and imaginary parts of $\phi_{c}$ are related to Bragg scattering coefficients and $\beta$.

It must be underlined that the $\mathrm{X}$-Bragg scattering model is not able to predict polarimetric scattering from natural surfaces characterized by large depolarizing properties as oil slick-covered sea surface [11], [16], [27].

\section{NUMERICAL SIMULATIONS}

In this section, experiments are undertaken to predict the behavior of $\sigma_{\phi_{c}}$ over slick-free sea surface according to the polarimetric model presented in section II.

First, the $\mathbf{X}$-Bragg covariance matrix $\mathbf{C}_{\mathbf{X}}$ is simulated according to eqs. (2) - (3) in the AOI range $20^{\circ}-50^{\circ}$. Reference is made to the $\mathrm{X}$-band and different $\beta$ values are considered. Although the latter is a global roughness parameter that depends on both local wind and non-local wind induced roughness, in this study we assume that $\beta$ depends on wind only since, at this observing scale, it is the main roughness driver.

To analyze the depolarization associated with the predicted $\mathbf{C}_{\mathbf{X}}$ matrix, the Cloude-Pottier entropy proposed in [29] is evaluated versus $\mathrm{AOI}$ for $\beta=5^{\circ}, 10^{\circ}$ and $20^{\circ}$, see Fig. 1(a), where they are depicted in blue, dashed red and dotted black curves, respectively. Those $\beta$ values describe flat, slightly rough and moderately rough sea surface, respectively. It can noted that the model allows describing a slightly depolarizing sea surface scattering, i. e., entropy values do not exceed 0.25 for any $\beta$ value. The plots in Fig. 1 (a) show that, even though an increasing in surface roughness results in larger entropy values, the depolarization effects predicted by X-Bragg model, as expected, are rather limited. This further witnesses, as pointed out in section II, that the X-Bragg model does not allow predicting polarimetric scattering from surfaces characterized by significant departure from Bragg-like scattering mechanism, i. e., oil slicks.

Once the depolarization properties of the model have been described using the Cloude-Pottier entropy, the behavior of the co-polarized phase difference can be analyzed. First, $\phi_{c}$ is predicted using (5), then its standard deviation is estimated over sea surface using a Montecarlo approach where $N=1000$ independent simulations are considered for each AOI. For each simulation, $\beta$ is randomly selected in the range $-30^{\circ}-+30^{\circ}$ according 


$$
\begin{gathered}
\mathbf{U}=\frac{1}{\sqrt{2}}\left(\begin{array}{ccc}
1 & 0 & 1 \\
1 & 0 & -1 \\
0 & \sqrt{2} & 0
\end{array}\right) \quad, \quad \mathbf{T}_{\mathbf{X}}=\left(\begin{array}{ccc}
\left|B_{c p}\right|^{2} & B_{c m} B_{c p}{ }^{*} s_{2 \beta} & 0 \\
B_{c p} B_{c m}{ }^{*} s_{2 \beta} & \frac{1}{2}\left|B_{c m}\right|^{2}\left(1+s_{4 \beta}\right) & 0 \\
0 & 0 & \frac{1}{2}\left|B_{c m}\right|^{2}\left(1-s_{4 \beta}\right)
\end{array}\right) \\
\phi_{c}=\angle\left(S_{h h} S_{v v}{ }^{*}\right)=\angle C_{X_{13}}=\angle\left(\frac{1}{2}\left|B_{c p}\right|^{2}-\frac{1}{4}\left|B_{c m}\right|^{2}\left(1-s_{4 \beta}\right)-j \Im\left(B_{c p} B_{c m}{ }^{*}\right) s_{2 \beta}\right)
\end{gathered} .
$$

to a uniform pdf.

Simulation results are shown in Fig. 1 (b), where $\sigma_{\phi_{c}}$ is depicted with respect to AOI. It can be noted that $\sigma_{\phi_{c}}$ increases non-linearly when increasing AOI, with the largest increase at AOIs larger than $35^{\circ} . \sigma_{\phi_{c}}$ values are always below $1.4^{\circ}$. This result is physically justified by the fact that a low-depolarizing model is implemented in a noise-free condition, e. g.; the sensor's noise floor is not accounted for. Hence, from a theoretical viewpoint, $p_{\phi_{c}}\left(\phi_{c}\right)$ should resemble a Dirac delta function, i. e., $\sigma_{\phi_{c}}=0^{\circ}$. Note, however, that Fig. 1 (b) points out that $\sigma_{\phi_{c}}$ increases with AOI. In fact, the relative broadening of $p_{\phi_{c}}\left(\phi_{c}\right)$, i. e., $\sigma_{\phi_{c}} / \bar{\phi}_{c}$, is around $50 \%$.
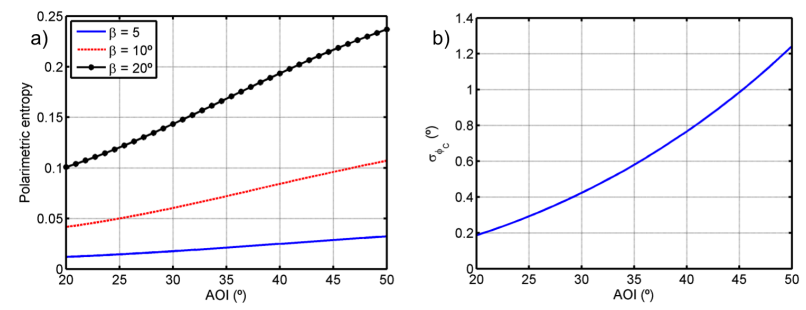

Fig. 1. Simulations based on the $\mathrm{X}$-Bragg model: (a) polarimetric entropy versus AOI for $\beta=5^{\circ}, 10^{\circ}$ and $20^{\circ}$, see blue, dashed red and dotted black curves, respectively; (b) $\sigma_{\phi_{c}}$ versus AOI.

\section{STUdy AREA AND SAR DATASET}

The study area is located in the northern part of the Gulf of Mexico, about $20 \mathrm{~km}$ off the Louisiana coast (see Fig. 2). The area is very rich in oil and gas fields and it also hosted one of the largest oil spill in the US history, i. e., the British Petroleum Deepwater Horizon platform blowout. Hence, this area has been continuosly monitored by different remote sensing platforms including the German Aerospace Center one that collected satellite TSX SAR imagery in dual-polarimetric HH-VV StripMap mode. The oil slicks, almost permanently observed in this area, are due to the 22 underwater oil wells of the Taylor Energy platform $\left(28.938^{\circ} \mathrm{N}, 88.971^{\circ} \mathrm{W}\right)$ that have been leaking an estimated amount of $100-$ 400 oil gallons per day since 16 September 2004, when the Hurricane Ivan has toppled several platforms in the Gulf of Mexico [26]. It was also estimated an average

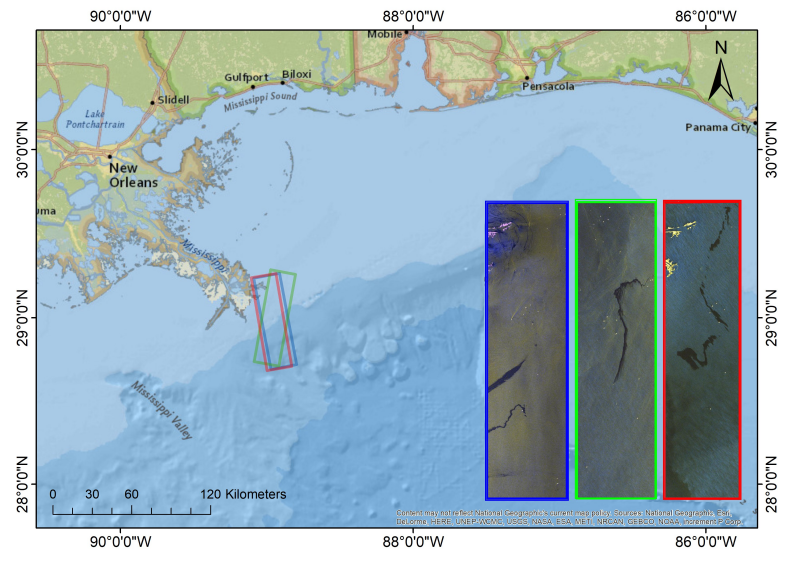

Fig. 2. Map of the study area, which is augmented by the footprints and the corresponding Pauli RGB images related to three TSX scenes. The Pauli images clearly show features, darker than the background sea, that are associated with the oil slicks.

slick thickness of $1 \mu \mathrm{m}$ and a half-life on the sea surface of 3-4 days [26]. Hence, those persistent oil slicks observed by the TSX mission for a long time represent an unprecedented opportunity to have a consistent and reliable dataset that can be exploited to better understand SAR-based sea oil slick monitoring.

In this study, a time series of TSX SAR imagery is considered that consists of 33 coherent HH-VV Level 1B Single Look Complex (SLC) SAR data acquired between July 2011 and April 2016 (see Table I). They are collected at different AOIs (in the range $25^{\circ}-45^{\circ}$ ) under low-to-moderate wind conditions (wind speed in the range $1.5-8 \mathrm{~m} / \mathrm{s}$ ). Wind speed information is retrieved from "42040" $\left(29.208^{\circ} \mathrm{N}, 88.226^{\circ} \mathrm{W}\right)$ and "Pill1" $\left(29.179^{\circ} \mathrm{N}, 89.259^{\circ} \mathrm{W}\right)$ buoy measurements and scatterometer-based wind field data freely available in [32] and [33], respectively. A general overview of the SAR dataset is provided in Table I. Note that the available SAR dataset limits our analysis on the influence of wind speed on $\sigma_{\phi_{c}}$ to moderate wind conditions (maximum wind speed observed in the SAR dataset is about $8.0 \mathrm{~m} / \mathrm{s}$, see Table I). However, a first attempt to analyze the oil slick detectability by means of polarimetric SAR features under higher wind conditions, 
TABLE I

OVERVIEW OF THE TSX SAR DATASET.

\begin{tabular}{|c|c|c|c|c|}
\hline SAR sensor, frequency $(\mathrm{GHz})$ & \multicolumn{4}{|c|}{ TerraSAR-X, 9.6} \\
\hline Imaging mode & \multicolumn{4}{|c|}{ StripMap coherent HH-VV } \\
\hline Number of SAR scenes & \multicolumn{4}{|c|}{33} \\
\hline Resolution (m) & \multicolumn{4}{|c|}{$1.2 \times 6.6$ (slant range $\times$ azimuth) } \\
\hline AOI range $\left(^{\circ}\right)$ & \multicolumn{4}{|c|}{$25-45$} \\
\hline Wind speed range $(\mathrm{m} / \mathrm{s})$ & \multicolumn{4}{|c|}{$2-8$} \\
\hline Study area & \multicolumn{4}{|c|}{ Gulf of Mexico, off Mississippi river delta } \\
\hline Observation period & \multicolumn{4}{|c|}{$1 / 7 / 2011-30 / 4 / 2016$} \\
\hline AOI-based dataset partitioning & Subset 1 & & Subset 2 & Subset 3 \\
\hline Average $\mathrm{AOI}\left({ }^{\circ}\right)$ & 26 & & 34 & 43 \\
\hline Number of SAR scenes & 10 & & 13 & 10 \\
\hline Average NESZ - HH (dB) & -23.0 & & -22.1 & -20.9 \\
\hline Average NESZ - VV (dB) & -23.2 & & -22.1 & -20.6 \\
\hline Wind speed range $(\mathrm{m} / \mathrm{s})$ & $2.3-7.8$ & & $3.0-7.6$ & $3-6.9$ \\
\hline Data Collection period & $1 / 7 / 2011-30 / 6 / 2014$ & $1 / 9 / 2$ & $011-30 / 5 / 2014$ & $1 / 4 / 2013-30 / 4 / 2016$ \\
\hline Wind-based dataset partitioning & \multicolumn{3}{|l|}{ Subset 4} & Subset 5 \\
\hline Wind regime & \multicolumn{2}{|c|}{ Low (wind speed $\leq 3.5 \mathrm{~m} / \mathrm{s}$ ) } & \multicolumn{2}{|c|}{ Moderate (wind speed $\geq 5 \mathrm{~m} / \mathrm{s}$ ) } \\
\hline Number of SAR scenes & \multicolumn{2}{|l|}{12} & \multicolumn{2}{|r|}{21} \\
\hline Average NESZ - HH (dB) & \multirow{2}{*}{\multicolumn{2}{|c|}{$\begin{array}{l}-21.9 \\
-21.9\end{array}$}} & & -21.9 \\
\hline Average NESZ - VV (dB) & & & \multicolumn{2}{|r|}{$\frac{-21.9}{25-45}$} \\
\hline AOI range $\left(^{\circ}\right)$ & \multicolumn{2}{|c|}{$25-45$} & & $25-45$ \\
\hline Data collection period & \multicolumn{2}{|c|}{$1 / 4 / 2012-30 / 4 / 2016$} & \multicolumn{2}{|c|}{$1 / 7 / 2011-30 / 3 / 2016$} \\
\hline
\end{tabular}

i. e., wind speed about $11.0 \mathrm{~m} / \mathrm{s}$, was undertaken in [34], where the performance was found to worsen with increasing wind speed.

The footprint of three SAR scenes is overlaid on the study area map together with the corresponding falsecolor RGB images (see Fig. 2). Note that the area affected by the oil seep is clearly visible in the three images and results in patches darker than the surrounding sea. Excerpts of the multi-polarization feature images evaluated over the same area affected by oil slicks in different dates, AOIs and sea state conditions are shown in Fig. 3, where six different SAR scenes randomly selected from the whole TSX time-series are considered (see Table I).

Examples of the SAR dataset are shown in Fig. 3 where six different SAR scenes, randomly selected from the whole TSX time-series (see Table I), are considered to cover the different AOIs, i. e., $26^{\circ}, 34^{\circ}$ and $43^{\circ}$, and both low and moderate wind conditions. They were collected on 23/07/2012, 25/04/2012, 22/04/2016, 29/05/2012, 02/08/2012 and 08/05/2014, see Fig. 3 (a) - (f). Geocoded images related to a $2.5 \mathrm{~km} \times 13.5 \mathrm{~km}$ area excerpted from six SAR scenes where the oil slick is imaged under different AOIs and wind conditions. The image is organized in matrix format. Columns refer to $\operatorname{AOI}\left(26^{\circ}, 34^{\circ}\right.$ and $43^{\circ}$, respectively), while the first two rows refer to $\sigma_{V V}^{0}$ images collected in low ((a) - (c)) and moderate $((\mathrm{d})-(\mathrm{f}))$ wind regime. The last two rows refer to $\sigma_{\phi_{c}}$ images evaluated over the corresponding SAR scenes shown in Fig. 3 (a) - (f).

\section{EXPERIMENTS ON ACTUAL SAR DATA}

In this section, experiments undertaken on actual SAR data to analyze the sensitivity of $\sigma_{\phi_{c}}$ to wind speed and SAR acquisition parameters (e. g., noise and AOI) are presented. The first experiment aims at evaluating the influence of noise and AOI, while the second one aims at investigating the effects of wind speed.

To perform this sensitivity analysis, the whole dataset is partitioned with respect to the parameters under investigation. The effects of noise and AOI are analyzed grouping SAR imagery into three subsets, which are classified as low, medium and large AOI $\left(26^{\circ}, 34^{\circ}\right.$ and $43^{\circ}$, see subsets $1-3$ in Table I, respectively); while the effects of wind speed are investigated partitioning the SAR dataset into two subsets, where low and moderate wind regimes are identified, i. e., wind speed lower than $3.5 \mathrm{~m} / \mathrm{s}$ and larger than $5 \mathrm{~m} / \mathrm{s}$ (see subsets 4 and 5 in Table I, respectively). Further details are listed in Table I. For each SLC SAR scene, the $\sigma_{\phi_{c}}$ is estimated using a $9 \times 9$ sliding window. Note that, since $\sigma_{\phi_{c}}$ is an unbiased estimator with respect to the window size, this window size is selected to guarantee that spatial resolution is almost preserved and a sufficient amount of samples are available to compute reliable statistics [18], [35]. Then the mean and standard deviation values of $\sigma_{\phi_{c}}$ are evaluated over 1000 samples randomly selected within two regions of interest (ROIs) belonging to slickfree and oil-covered sea surface. Note that, for reference purposes, $\sigma_{V V}^{0}$ is also analyzed using the same slick-free and oil-covered ROIs defined for $\sigma_{\phi_{c}}$.

The first experiment consists of analyzing the broadening 
a)

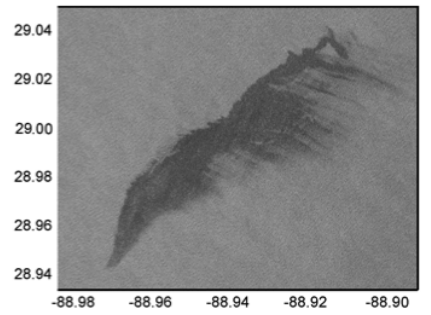

d)

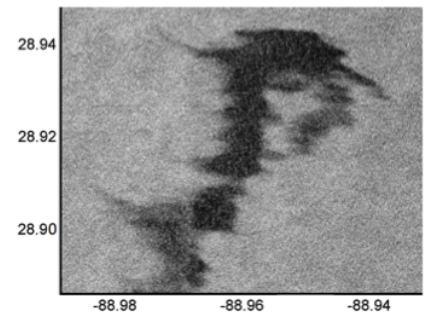

g)

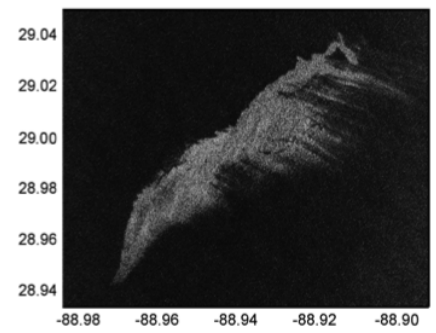

j)

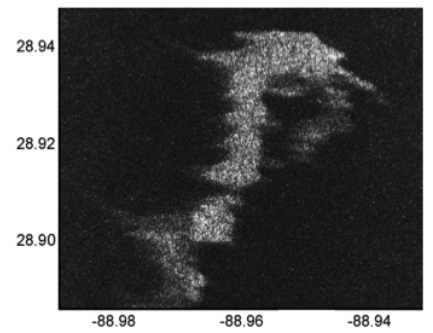

b)

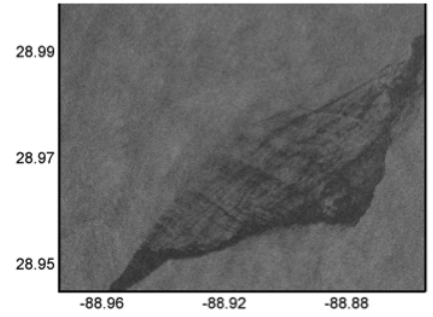

e)

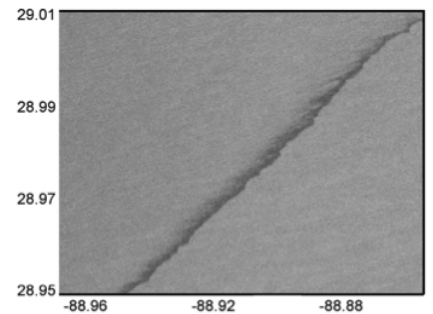

h)

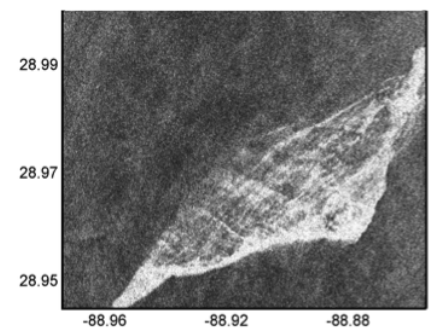

k)

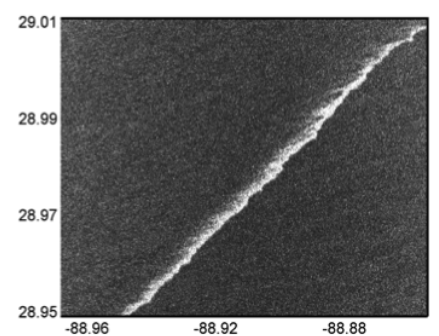

c)

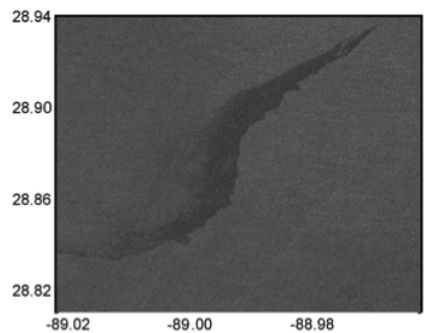

f)

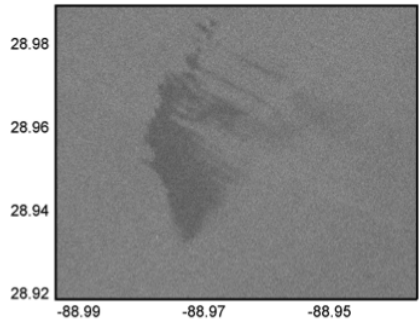

i)

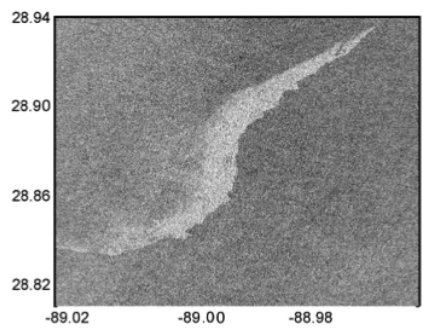

I)

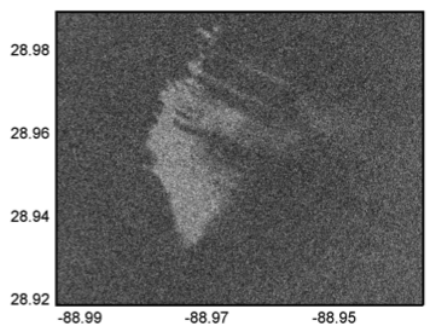

Fig. 3. Geocoded images related to a $2.5 \mathrm{~km} \times 13.5 \mathrm{~km}$ area excerpted from six SAR scenes where the oil slick is imaged under different AOIs and wind conditions. The image is organized in matrix format. Columns refer to AOI $\left(26^{\circ}, 34^{\circ}\right.$ and $43^{\circ}$, respectively), while the first two rows refer to $\sigma_{V V}^{0}$ images collected in low ((a) - (c)) and moderate ((d) - (f)) wind regime. They were collected on 23/07/2012, 25/04/2012, 22/04/2016, 29/05/2012, 02/08/2012 and 08/05/2014, respectively. (g) - (l) refer to $\sigma_{\phi_{c}}$ images evaluated over the corresponding SAR scenes shown in (a) - (f).

of the $p_{\phi_{c}}\left(\phi_{c}\right)$ induced by noise, i. e., the sensor's NESZ [36], and by scene-induced depolarization (see Fig. 4 (a), where $\sigma_{\phi_{c}}$ data-points evaluated within the slick-free and oil-covered ROIs are shown). The depolarization induced by the scene carries on information on the scattering properties of the observed scene; while NESZ affects the polarimetric properties of the scattered wave introducing depolarization in the backscattered signal when it is close to (or below) NESZ. Hence, the effect of both NESZ and scene depolarization depend on the presence of surfactants.

When dealing with slick-free sea surface, see Fig. 4 (a), one can note that $\sigma_{\phi_{c}}$ values increase with AOI. This trend agrees with model's predictions discussed in section III (see Fig. 1 (b)). Nonetheless, predicted $\sigma_{\phi_{c}}$ values, as expected, underestimate actual measurements mainly due to the presence of noise that affects actual SAR data. To distinguish effects related to NESZ from scene-induced depolarization, reference is made to single-polarization backscattering, see Fig. 4 (b), where data-points related to $\sigma_{V V}^{0}$ evaluated over slick-free and oil-covered ROIs are shown together with NESZ. One can see that slick-free data-points are well-above NESZ at low and intermediate AOIs; while they approach NESZ at larger AOIs. This implies that NESZ and sceneinduced depolarization start playing a role at AOI larger than $34^{\circ}$. NESZ is the key broadening factor at $\mathrm{AOI} \approx$ $43^{\circ}$. In fact, the estimated NESZ ranges from about -24 

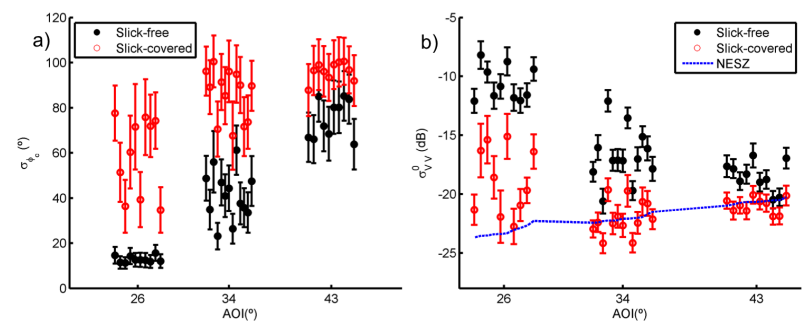

Fig. 4. Mean and standard deviation values of: (a) $\sigma_{\phi_{c}}$ and (b) $\sigma_{V V}^{0}$ evaluated within slick-free (black) and oiled (red) ROIs excerpted from the SAR data subsets $1-3$, see Table I. In sub-figure (b) the NESZ profile estimated from actual SAR measurements is also annotated as a dashed blue line.

$\mathrm{dB}$ (at $\mathrm{AOI}=26^{\circ}$ ) up to approximately $-21 \mathrm{~dB}$ (at AOI $\left.=43^{\circ}\right)$, see Fig.4 (b).

When dealing with oil-covered sea surface, see Fig. 4 (a), $\sigma_{\phi_{c}}$ values still exhibit an increasing trend with AOI. According to Fig. 4 (b), one can see that the effect of NESZ is non-negligible for almost all the data-points related to the oil-covered sea surface, with the smallest effect at $\mathrm{AOI} \approx 26^{\circ}$. As a result, the large $\sigma_{\phi_{c}}$ values related to oil-covered sea surface at AOI $\approx 26^{\circ}$ are mostly due to the depolarizing behavior of the scattering from the oil slick; while at larger AOIs, both NESZ and scene-induced depolarization play a role. Hence, in the case of slick-covered sea surface, experimental results suggest that the noise significantly contributes to smooth the increasing trend of $\sigma_{\phi_{c}}$ with respect of AOI. In fact, $\sigma_{\phi_{c}}$ tends to saturate at AOI larger than $34^{\circ}$.

It can be noted that $\sigma_{\phi_{c}}$ values are always wellseparated from the slick-free ones (Fig. 4 (a)). This implies that oil can be always distinguished from the background sea over the whole AOI range. Nevertheless, the oil/sea separation decreases with AOI. Hence, the broadening of oil $p_{\phi_{c}}\left(\phi_{c}\right)$ is due to two concurring factors: the large depolarization resulting from oil's backscattering and the NESZ. In particular, at AOI larger than $26^{\circ}$, NESZ affects also slick-free sea surface scattering resulting in $\sigma_{\phi_{c}}$ values larger than the ones at $26^{\circ}$. This justifies the reduction of oil/sea separation at larger AOIs .

To quantitatively discuss the broadening of $p_{\phi_{c}}\left(\phi_{c}\right)$ with respect to AOI, the errorbars of Fig. 4 are analyzed in Table II where mean and standard deviation values of $\sigma_{\phi_{c}}$ errorbars are listed together with errorbar values related to $\sigma_{V V}^{0}$ and $\sigma_{H H}^{0}$. In addition, a new figure of merit is also proposed, $\Delta$, to measure the sensitivity of $\sigma_{\phi_{c}}$ to the scene variability. $\Delta$, defined as the ratio between standard deviation and the mean value, is listed in Table II. Hence, it can be considered as a relative measure of the standard deviation that is mostly related to the variability of the scene observed processing the time series.
When dealing with slick-free sea surface, $\sigma_{\phi_{c}}$ mean value approximately triples from $26^{\circ}$ to $34^{\circ}$, while it doubles from $34^{\circ}$ to $43^{\circ}$. This is mostly due to the effects of NESZ that starts to play a non-negligible role at AOI $>34^{\circ}$. In terms of sensitivity to the scene variability, $\sigma_{\phi_{c}}$ exhibits a remarkable sensitivity to sea state conditions at $\mathrm{AOI} \leq 34^{\circ}$, i. e., $\Delta \approx 22 \%$. This sensitivity significantly decreases at AOI $>34^{\circ}$ likely due to the fact that, as suggested by the results of the noise analysis, noise sources dominate and, therefore, very limited sensitivity to actual sea state conditions is observed $(\Delta=15 \%)$. In addition, results listed in Table II witness that the $\sigma_{\phi_{c}}$ sensitivity to the scene variability is significantly larger than the one exhibited by $\sigma_{V V}^{0}$ and $\sigma_{H H}^{0}$, i. e., $\Delta \approx 22 \%$ and $\approx 7 \%$ (at $\mathrm{AOI} \leq 34^{\circ}$ ), respectively.

When dealing with oil-covered sea surface, $\sigma_{\phi_{c}}$ mean value increases slightly with $\mathrm{AOI}$, i. e., from $59^{\circ}$ at $\mathrm{AOI}=26^{\circ}$ up to $96^{\circ}$ at $\mathrm{AOI}=43^{\circ}$. This confirms the saturation of $\sigma_{\phi_{c}}$ at $\mathrm{AOI} \geq 34^{\circ}$ due to the concurring effects of NESZ and scene depolarization in the broadening of $p_{\phi_{c}}\left(\phi_{c}\right)$. However, $\sigma_{\phi_{c}}$ mean values are wellabove the corresponding values assumed over slick-free sea surface for all the AOIs. In terms of sensitivity to the scene variability, $\sigma_{\phi_{c}}$ exhibits a pronounced sensitivity to the surfactant at AOI $<34^{\circ}$, i. e., $\Delta=24 \%$. Even though $\sigma_{\phi_{c}}$ evaluated over oil-covered sea surface at AOI $=26^{\circ}$ calls for almost the same $\Delta$ value of the slick-free case, its sensitivity starts to significantly decrease at AOI > $26^{\circ}$. This is mostly due to NESZ that contaminates the signal scattered-off the scene and, thus, reducing the sensitivity of $\sigma_{\phi_{c}}$ to the surfactant properties. Nevertheless, even in this case, results listed in Table II show that the sensitivity of $\sigma_{\phi_{c}}$ to the scene variability is more pronounced than the one that characterizes $\sigma_{V V}^{0}$ and $\sigma_{H H}^{0}$, i. e., $\Delta=22 \%$ and $\approx 9 \%$ (at $\mathrm{AOI}<34^{\circ}$ ), respectively.

The second experiment consists of analyzing the effect of wind speed on the broadening of $p_{\sigma_{\phi_{c}}}\left(\sigma_{\phi_{c}}\right)$, see Fig. 5 , where $\sigma_{\phi_{c}}$ data-points evaluated within slick-free (Fig. 5 (a)) and oil-covered (Fig. 5 (b)) ROIs are shown. Mean and standard deviation values of $\sigma_{\phi_{c}}$ for different AOIs are depicted, see black dots $\left(26^{\circ}\right)$, blue dots $\left(34^{\circ}\right)$ and red crosses $\left(43^{\circ}\right)$. This analysis is undertaken on the SAR subsets 4 and 5, see Table I.

When dealing with slick-free sea surface, no clear trend can be observed for $\sigma_{\phi_{c}}$ data-points belonging to low ( $\leq$ $3.5 \mathrm{~m} / \mathrm{s})$ and moderate $(\geq 5 \mathrm{~m} / \mathrm{s})$ wind regimes sampled at the three AOIs, see Fig. 5 (a). To quantitatively confirm this behavior, the mean values of $\sigma_{\phi_{c}}$ errorbars and $\Delta$ values are listed in Table III together with reference $\sigma_{V V}^{0}$ and $\sigma_{H H}^{0}$ values. One can note that $\sigma_{\phi_{c}}=43^{\circ}$ for both wind regimes. This witnesses that, under low-tomoderate regimes, wind speed variability has a negligible impact on the broadening of $p_{\sigma_{\phi_{c}}}\left(\sigma_{\phi_{c}}\right)$. No clear trend with respect to wind speed and no remarkable difference 
TABLE II

MEAN, STANDARd DEVIATION (STD) AND AVERAGE RELATIVE VARIABILITy $(\Delta)$ RELATED TO THE ERRORBARS OF Fig. 4. Note THAT RESULTS ARE ALSO LISTED FOR $\sigma_{H H}^{0}$ THAT IS NOT SHOWN IN FIG. 4 TO SAVE SPACE.

\begin{tabular}{|c|c|c|c|c|c|c|c|c|c|c|}
\hline \multirow{2}{*}{ ROI } & Feature & \multicolumn{4}{|c|}{$\sigma_{\phi_{c}}\left({ }^{\circ}\right)$} & \multicolumn{3}{|c|}{$\sigma_{V V}^{0}(\mathrm{~dB})$} & \multicolumn{3}{|c|}{$\sigma_{H H}^{O}(\mathrm{~dB})$} \\
\cline { 2 - 13 } & $\mathrm{AOI}\left({ }^{\circ}\right)$ & 26 & 34 & 43 & 26 & 34 & 43 & 26 & 34 & 43 \\
\hline \hline \multirow{3}{*}{ Sea surface } & mean & 13 & 41 & 75 & -10.6 & -16.7 & -18.5 & -10.8 & -18.0 & -20.1 \\
\cline { 2 - 13 } & std & \pm 3 & \pm 9 & \pm 11 & \pm 1.1 & \pm 0.9 & \pm 0.8 & \pm 1.1 & \pm 0.9 & \pm 0.8 \\
\cline { 2 - 12 } & $\Delta(\%)$ & 23 & 22 & 15 & 9 & 5 & 4 & 10 & 5 & 4 \\
\hline \hline \multirow{3}{*}{ Oil slick } & mean & 59 & 86 & 96 & -18.8 & -22.0 & -21.0 & -19.2 & -22.2 & -21.5 \\
\cline { 2 - 11 } & std & \pm 14 & \pm 12 & \pm 11 & \pm 1.7 & \pm 1.0 & \pm 0.7 & \pm 1.9 & \pm 0.9 & \pm 0.7 \\
\cline { 2 - 11 } & $\Delta(\%)$ & 24 & 14 & 11 & 9 & 5 & 3 & 10 & 4 & 3 \\
\hline
\end{tabular}

between low and moderate wind regimes are observed even when $\sigma_{V V}^{0}$ and $\sigma_{H H}^{0}$ are considered, see Fig. 5 (c) and Table III. In terms of scene variability, $\sigma_{\phi_{c}}$ calls for a significant sensitivity to sea state conditions, i. e., $\Delta=19 \%$, for both wind regimes, witnessing that the sensitivity of $\sigma_{\phi_{c}}$ to actual sea state does not depend on wind speed. Results listed in Table III also show that, again, $\sigma_{\phi_{c}}$ is more sensitive to scene variability if compared to $\sigma_{V V}^{0}$ and $\sigma_{H H}^{0}(\Delta=19 \%$ and $\approx 6 \%$, respectively).

When dealing with slick-covered sea surface, the behavior of $\sigma_{\phi_{c}}$, evaluated under low and moderate wind regimes, does not exhibit a particular trend, see Fig. 5 (b). This is quantitatively confirmed by the mean values of $\sigma_{\phi_{c}}$ errorbars and $\Delta$ values listed in Table III together with reference $\sigma_{V V}^{0}$ and $\sigma_{H H}^{0}$ values. It can be noted that $\sigma_{\phi_{c}} \approx 81^{\circ}$ for both wind regimes, i. e., they are almost doubled with respect to the ones measured over the sea surface ROI but they do not significantly depend on wind speed. $\sigma_{V V}^{0}$ and $\sigma_{H H}^{0}$ behave similar to the slick-free case, see Fig. 5 (d) and Table III. In terms of scene variability, $\sigma_{\varphi_{c}}$ is characterized by $\Delta \approx 16 \%$ for both wind regimes that results in a more pronounced sensitivity on actual oil conditions with respect to $\sigma_{V V}^{0} / \sigma_{H H}^{0}$ (whose $\Delta$ values are approximately $6 \%$ ). Nonetheless, it can be noted that the $\sigma_{\phi_{c}}$ sensitivity to scene variability slightly reduces with respect to the slick-free case. This is likely due to the fact that the presence of a strong-damping oil layer over the sea surface attenuates the roughness induced by local wind.

The availability of DP co-polarized SAR measurements make possible extracting the above-discussed copolarized phase difference and the so-called Pauli phase, i. e., the argument of $\mathrm{HH}+\mathrm{VV} / \mathrm{HH}-\mathrm{VV}$, whose statistic is given in [27]. The latter consists of information on the dielectric properties of the observed scene; hence, for sake of completeness, we accomplished a further study that aimed at analyzing the sensitivity of the Pauli phase to sea oil seeps. Both the Pauli phase and its variabiloty, measured by the standard deviation, are evaluated. Results related to a meaningful test case are shown in Fig. 6, where the $\sigma_{V V}^{0}$ of the SAR scene (a)
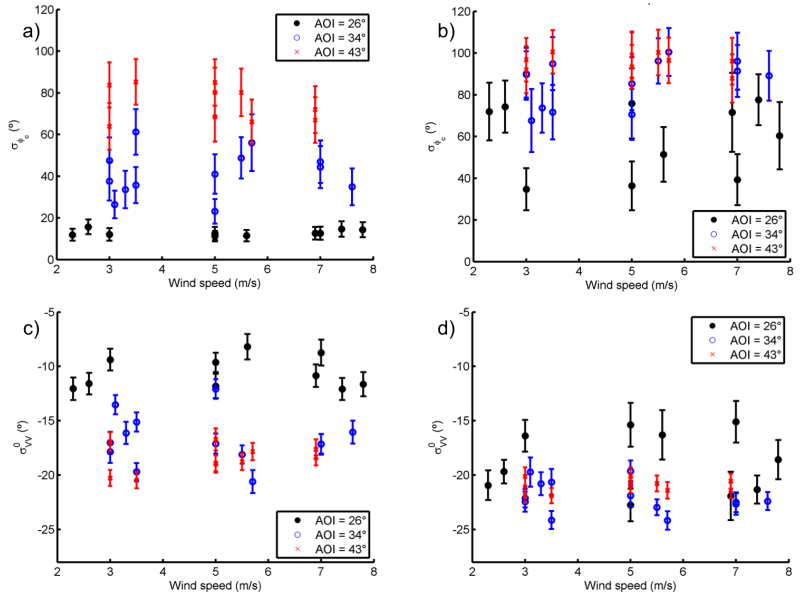

Fig. 5. Sensitivity analysis with respect to wind speed. (a) - (b): mean and standard devation values of $\sigma_{\phi_{c}}$ evaluated over slick-free and oil-covered sea surface ROIs, respectively; (c) - (d) mean and standard devation values of $\sigma_{V V}^{0}$, evaluated over slick-free and oilcovered sea surface ROIs, respectively. Data-points belonging to the different AOIs are depicted in black dots $\left(26^{\circ}\right)$, blue circles $\left(34^{\circ}\right)$ and red crosses $\left(43^{\circ}\right)$, respectively.

is shown together with the Pauli phase and its standard deviation (b)-(c) and the co-polarized phase difference with its standard deviation (d)-(e). It can be noted that $\sigma_{\phi_{c}}$ success in highlighting the oil-affected area while neither the Pauli phase, nor its standard deviation show any significant hint related to the oil seep. This is likely due to the fact that the Pauli phase is related to the dielectric properties of the scattering scene that, as it was demonstrated in [37], play a marginal role when dealing with sea oil seep backscattering.

\section{CONCLUSIONS}

In this study, a time series of 33 TSX HH-VV StripMap mode SAR images collected over the wellknown Taylor Energy oil seep in the Gulf of Mexico in a broad range of AOIs and wind speeds, is exploited to investigate the sensitivity of $\sigma_{\phi_{c}}$ to both SAR acquisition parameters (e. g., AOI and NESZ) and meteo-marine 
TABLE III

MEAN, STANDARD DEVIATION (STD) AND AVERAGE RELATIVE VARIABILITY $(\Delta)$ VALUES WITH RESPECT TO WIND REGIME RELATED TO $\sigma_{\phi_{c}}, \sigma_{V V}^{0}$ AND $\sigma_{H H}^{0}$ EVALUATED OVER SLICK-FREE AND OIL SLICK-COVERED ROIS.

\begin{tabular}{|c|c|c|c|c|c|c|c|}
\hline \multirow{2}{*}{ ROI } & Feature & \multicolumn{2}{|c|}{$\sigma_{\phi_{c}}\left(^{\circ}\right)$} & \multicolumn{2}{|c|}{$\sigma_{V V}^{0}(\mathrm{~dB})$} & \multicolumn{2}{c|}{$\sigma_{H H}^{0}(\mathrm{~dB})$} \\
\cline { 2 - 8 } & Wind Regime & Low & Moderate & Low & Moderate & Low & Moderate \\
\hline \hline \multirow{3}{*}{ Sea surface } & mean & 43 & 43 & -15.8 & -15.2 & -16.8 & -16.3 \\
\cline { 2 - 8 } & std & \pm 8 & \pm 8 & \pm 0.9 & \pm 1.0 & \pm 0.9 & \pm 1.0 \\
\cline { 2 - 8 } & $\Delta(\%)$ & 19 & 19 & 6 & 7 & 5 & 6 \\
\hline \hline \multirow{3}{*}{ Oil slick } & mean & 80 & 82 & -20.9 & -20.6 & -21.3 & -21.0 \\
\cline { 2 - 8 } & std & \pm 12 & \pm 13 & \pm 1.0 & \pm 1.2 & \pm 1.0 & \pm 1.2 \\
\cline { 2 - 8 } & $\Delta(\%)$ & 15 & 16 & 5 & 6 & 5 & 6 \\
\hline
\end{tabular}

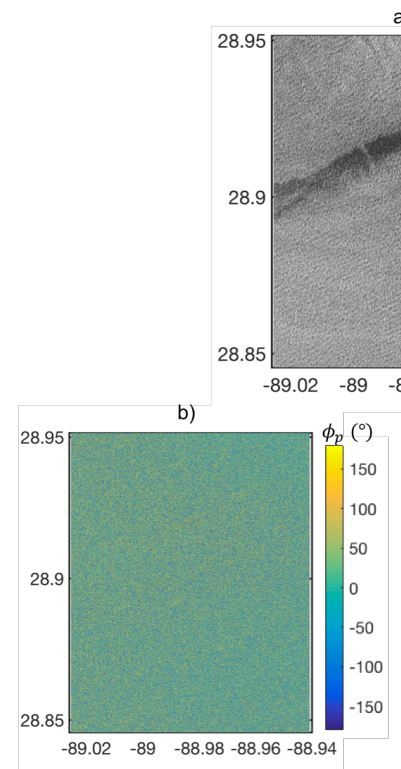

a)
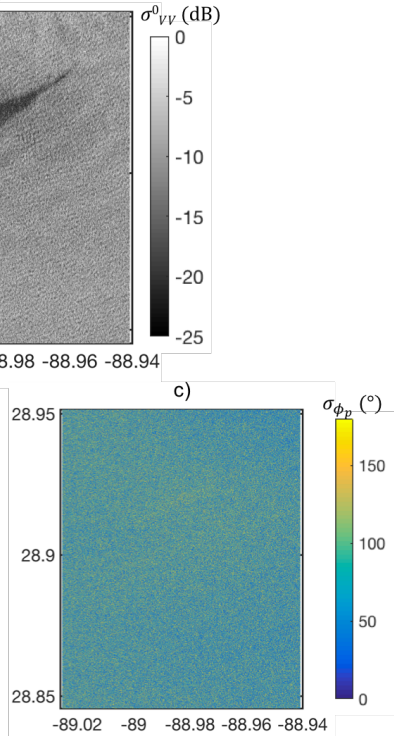

d)
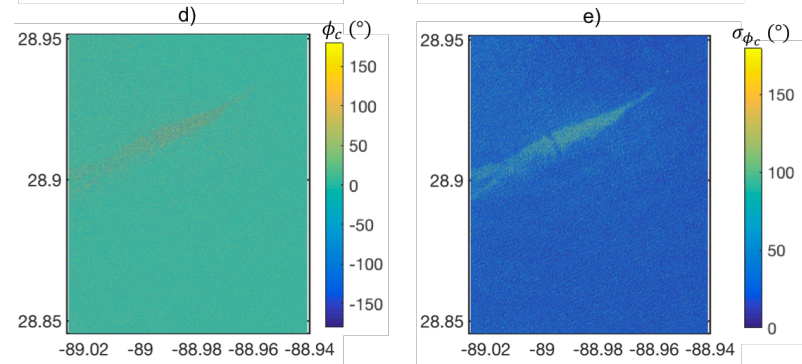

Fig. 6. Co-polarized phase difference, $\phi_{c}$, versus Pauli phase, $\phi_{p}$. (a): geocoded VV-polarized SAR images(in $\mathrm{dB}$ scale), where the sea oil slick is clearly visible. (b)-(c): Pauli phase and its standard deviation images, respectively, where no slick is observed; (d)-(e): co-polarized phase difference and its standard deviation images, respectively, where the presence of the oil slick is emphasized with respect to the surrounding sea.

conditions (e. g., wind speed, polarimetric properties of the observed scene). This SAR dataset offers, for the first time, the opportunity to analyze the behavior of $\sigma_{\phi_{c}}$ over both slick-free and slick-covered sea surface under a large variety of imaging parameters and scene conditions. The behavior of single-polarization intensity channels is also considered as a reference.

The main outcomes of this study are summarized as follows:

- The noise-free behavior of $\sigma_{\phi_{c}}$ with respect to AOI can be well-predicted by the X-Bragg scattering model;

- $\sigma_{\phi_{c}}$ increases when increasing AOI over both slickfree and oil-covered sea surface. The increasing trend is more pronounced in the slick-free case;

- The noise plays a dominant role in the broadening of $p_{\phi_{c}}\left(\phi_{c}\right)$ over both slick-free and slick-covered sea surface. The broadening of $p_{\phi_{c}}\left(\phi_{c}\right)$ at lower (larger) AOIs is mostly due to scene-induced depolarization (NESZ);

- Under low-to-moderate wind conditions, the effect of wind speed on the broadening of $p_{\phi_{c}}\left(\phi_{c}\right)$ is negligible. This is of relevant importance when dealing with sea oil slick detection;

- The $\sigma_{\phi_{c}}$ sensitivity to scene variability within the time series is more significant at lower AOIs since, at larger AOIs, data-points are contaminated by noise. This sensitivity is more pronounced than the $\sigma_{V V}^{0} / \sigma_{H H}^{0}$ one;

- Oil/sea $\sigma_{\phi_{c}}$ data-points are well separated at all the AOIs, with this separation that reduces with AOI mostly due to both oil-induced depolarization and NESZ;

- The so-called Pauli phase does not show any sensitivity with respect to sea oil slicks.

It is important to underline that those results may be useful to support the design of robust and effective polarimetric SAR-based algorithms for sea oil slick monitoring. 


\section{ACKNOWLEDGMENTS}

This study is partly funded by the Università degli Studi di Napoli Parthenope, project ID DING 202, by ANVUR under the project entitled "Fondo per le attività base di ricerca", and by the European Space Agency under the Dragon 4 project ID 32235. We thank the German Space Agency (DLR) who provided TerraSARX SAR data under the AO OCE1045 and the National Oceanic and Atmospheric Administration (NOAA) who provided buoy data.

\section{REFERENCES}

[1] M. F. Fingas, and C. E. Brown, "Handbook of Oil Spill Science and Technology, Chapter 12: Oil Spill Remote Sensing," Wiley, pp. 313-356, 2015.

[2] C. Brekke, and A. H. S. Solberg, "Oil spill detection by satellite remote sensing," Remote Sens. Env., vol. 95, no. 1, pp. 1-13, 2005.

[3] A. H. S. Solberg, "Remote sensing of ocean oil-spill pollution," Proceedings of the IEEE, vol. 100, no. 10, pp. 2931-2945, 2012.

[4] Y. Cheng, B. Liu, X. Li, F. Nunziata, Q. Xu, X. Ding, M. Migliaccio and W.G. Pichel, "Monitoring of oil spill trajectories with COSMO-skymed X-band SAR images and model simulation," IEEE J. Selec. Top. Appl. Earth Obs. Remote Sens., vol. 7, no. 7, pp. 2895-2901, 2014.

[5] A. Montuori, F. Nunziata, M. Migliaccio, and P. Sobieski, "XBand Two-Scale Sea Surface Scattering Model to Predict the Contrast due to an Oil slick," IEEE J. Sel. Topics Appl. Earth Observ. Remote Sens., vol. 9, no. 11, pp. 4970-4978, 2016.

[6] M. Gade, H. Huhnerfuss, and G. Korenowski, "Marine Surface Films, Chapter 1: Basic Physiochemical Principles of Monomolecular Sea Slicks and Crude Oil Spills," SpringerVerlag Berlin Heidelberg, pp. 21-35, 2006.

[7] X. Ding, F. Nunziata, X. Li, and M. Migliaccio, "Performance analysis and validation of waterline extraction approaches using single- and dual-polarimetric SAR data," IEEE J. Sel. Topics Appl. Earth Observ. Remote Sens., vol. 8, no. 3, pp. 1019-1027, 2015.

[8] F. Nunziata, A. Buono, M. Migliaccio, and G. Benassai, "DualPolarimetric C- and X-Band SAR Data for Coastline Extraction," IEEE J. Sel. Topics Appl. Earth Observ. Remote Sens., vol. 9, no. 11, pp. 4921-4928, 2016.

[9] F. Nunziata, M. Migliaccio, and C. E. Brown, "Reflection Symmetry for Polarimetric Observation of Man-Made Metallic Targets at Sea," IEEE J. Ocean. Eng., vol. 37, no. 3, pp. 384-394, 2012.

[10] D. Velotto, F. Nunziata, M. Migliaccio, and S. Lehner, "DualPolarimetric TerraSAR-X SAR Data for Target at Sea Observation," IEEE Geosci. Remote Sens. Lett., vol. 10, no. 5, pp. 1114-1118, 2013.

[11] M. Migliaccio, F. Nunziata, and A. Buono, "A review on SAR polarimetry for sea oil slick observation," Int. J. Remote Sens., vol. 36, no. 12, pp. 3243-3273, 2015.

[12] M. J. Collins, M. Denbina, B. Minchew, C. E. Jones, and B. Holt, "On the Use of Simulated Airborne Compact Polarimetric SAR for Characterizing Oil-Water Mixing of the Deepwater Horizon Oil Spill," IEEE J. Sel. Topics Appl. Earth Observ. Remote Sens., vol. 8, no. 3, pp. 1062-1077, 2015.

[13] B. Minchew, "Determining the mixing of oil and sea water using polarimetric synthetic aperture radar," Geophys. Res. Lett., vol. 39 , no. 16, pp. 1-6, 2012.

[14] M. Migliaccio and F. Nunziata, "On the exploitation of polarimetric SAR data to map damping properties of the Deepwater Horizon oil spill," Int. J. Remote Sens., vol. 35, no. 10, pp. 34993519, 2014.

[15] S. Skrunes, C. Brekke, T. Eltoft, and V. Kudryavtsev, "Comparing near-coincident $\mathrm{C}$ - and X-band SAR acquisitions of marine oil spills," IEEE Trans. Geosci. Remote Sens., vol. 53, no. 4, pp. 1958-1975, 2015.
[16] A. Buono, F. Nunziata, M. Migliaccio, and X. Li, "Polarimetric analysis of compact-polarimetry SAR architectures for sea oil slick observation," IEEE Trans. Geosci. Remote Sens., vol. 54, no. 10, pp. 5862-5874, 2016.

[17] S. Skrunes, C., Brekke, and A. P. Doulgeris, "Characterization of Low-Backscatter Ocean Features in Dual-Copolarization SAR Using Log-Cumulants," IEEE Geosci. Remote Sens. Lett., vol. 12, no. 4, pp. 836-840, 2015.

[18] D. Velotto, M., Migliaccio, F., Nunziata, and S. Lehner, "Dualpolarized TerraSAR-X Data for Oil Spill Observation," IEEE Trans. Geosci. Remote Sens., vol. 30, pp. 1587-1602, 2011.

[19] F. Nunziata, A. Gambardella, and M. Migliaccio, "A Unitary Mueller-based View of Polarimetric SAR Oil Slick Observation," Int. J. Remote Sens., vol. 33, no. 20, pp. 6403-6425, 2012.

[20] M. Migliaccio, F., Nunziata, and A. Gambardella, "On the Copolarised Phase Difference for Oil Spill Observation," Int. J. Remote Sens., vol. 30, pp. 1587-1602, 2009.

[21] K. Sarabandi, "Derivation of phase statistics from the Mueller matrix," Radio Sci., vol. 27, no. 5, pp. 553-560, 1992.

[22] I. R. Joughin, D. P. Winebrenner, and D. B. Percival, "Probability density functions for multilook polarimetric signatures," IEEE Trans. Geosci. Remote Sens., vol. 32, no. 3, pp. 562-574, 1994.

[23] D. L. Schuler, J. S. Lee, and K. W. Hoppel, "Polarimetric SAR image signatures of the ocean and Gulf Stream features," IEEE Trans. Geosci. Remote Sens., vol. 31, no. 6, pp. 1210-1221, 1993.

[24] J. S. Lee, K. W. Hoppel, S. A. Mango, and A. R. Miller, "Intensity and phase statistics of multilook polarimetric and interferometric SAR imagery," IEEE Trans. Geosci. Remote Sens., vol. 32, no. 5, pp. 1017-1028, 1994.

[25] A. Guissard, "Phase calibration of polarimetric radars from slightly rough surfaces," IEEE Trans. Geosci. Remote Sens., vol. 32, no. 3, pp. 712-715, 1994.

[26] Gulf Monitoring Consortium, "Report on Activities from April 2011 to October 2011," Tech. Rep., 2011.

[27] I. Hajnsek, E. Pottier, and S.R. Cloude, "Inversion of surface parameters from polarimetric SAR," IEEE Trans. Geosci. Remote Sens., vol. 41, no. 4, pp. 727-744, 2003.

[28] J.-S. Lee and E. Pottier, "Polarimetric radar imaging: from basics to application," CRC Press, Taylor \& Francis Group, 2009.

[29] S. R. Cloude and E. Pottier, "A Review of Target Decomposition Theorems in Radar Polarimetry," IEEE Trans. Geosci. Remote Sens., vol. 34, no. 2, pp. 498-518, 1996.

[30] J. Yin, J. Yang, Z.-S. Zhou, and J. Song, "The extended Bragg scattering model-based method for ship and oil-spill observation using compact polarimetric SAR," IEEE J. Sel. Topics Appl. Earth Observ. Remote Sens., vol. 8, no. 8, pp. 3760-3771, 2015.

[31] A. Buono, F. Nunziata, and M. Migliaccio, "Analysis of Full and Compact Polarimetric SAR Features Over the Sea Surface," IEEE Geosci. Remote Sens. Lett., vol. 13, no. 10, pp. 1527-1531, 2016.

[32] National Oceanic and Atmospheric Administration's National Data Buoy Center, accessed on 13 December 2017. [Online]. Available: http://www.ndbc.noaa.gov/.

[33] Remote Sensing System, accessed on 19 December 2017. [Online]. Available: http://www.remss.com/measurements/wind/.

[34] S. Skrunes, C. Brekke, C. E. Jones and B. Holt, "A Multisensor Comparison of Experimental Oil Spills in Polarimetric SAR for High Wind Conditions," IEEE J. Selec. Top. Appl. Earth Obs. Remote Sens., vol. 9, no. 11, pp. 4948-4961, 2016.

[35] R. Touzi, Ridha, A. Lopes, J. Bruniquel and P. W. Vachon, "Coherence Estimation for SAR Imagery," IEEE Trans. Geosci. Remote Sens., vol. 37, no. 1, pp. 135-149, 1999.

[36] J. C. Curlander and R. N. McDonough, "Synthetic Aperture Radar System and Signal Processing," John Wiley \& Sons, Jin Au Kong Editor, New York, 1990.

[37] F. Nunziata, C. R. de Macedo, A. Buono, D. Velotto, and M. Migliaccio, "On the analysis of a time series of X-band TerraSAR-X SAR imagery over oil seepages," Int. J. Remote Sens., in print., 2018. 